\title{
PERINATAL OUTCOME IN LOW AMMNIOTIC FLUID INDEX
}

1. Assistant Professor

Department of Gynae \& Obs. Independent University Hospital, Faisalabad

2. Associate Professor

Department of Gynae \& Obs. Independent University Hospital Faisalabad.

3. Assistant Professor Department of Gynae \& Obs Independent University Hospital, Faisalabad.

\section{Correspondence Address:}

Dr. Iram Aslam

Assistant Professor

Department of Gynae \& Obs.

Independent University Hospital, Faisalabad.

Article received on:

15/12/2014

Accepted for publication:

09/01/2015

Received after proof reading:

$15 / 01 / 2015$

Dr. Iram Aslam¹, Dr. Nazia Mussarat², Dr. Sadia Bano³

ABSTRACT: Introduction: In high risk pregnancies, there is a dire need to assess amniotic fluid volume. If values of amniotic fluid volume fall within normal limits, it reassures us regarding well being of fetus. The chronically stressed fetus is likely to have low amniotic fluid. Amniotic fluid index is a reliable fetal surveillance test and in some obstetrical units highly reliable parameter. Decreased amniotic fluid index has been considered as an indicator of poor neonatal outcome. AFI may be used to predict the occurrence of thick meconium stained liquor and need for intervention for fetal distress in post date pregnancies. Study Design: Descriptive Study. Settings: Obstetrics and Gynaecology department of Fatima Memorial Hospital, Lahore affiliated with Fatima College of Medicine \& dentistry Lahore. Duration: 6 month from $7 / 2 / 2007$ to $7 / 8 / 2007$. Subjects and Methods: All pregnant women, meeting the inclusion criteria admitted in labour room either through emergency or OPD were selected. The AFI was calculated ultrasonically and these patients were followed till the time of delivery and the APGAR scores of newborn were compared with AFI. Results: During the study period AFI was measured in 60 patients. Among these patients, 32 patients had AFI between 3-4 cm and babies were meconium stained, 23 patients had AFI of 5-6 cm and babies had normal APGAR at the time of delivery. AFI was $<2 \mathrm{~cm}$ in 5 patients and babies required resuscitation and admitted in nursery. Conclusion: The AFI is a reliable predictor of neonatal outcome.

Key words: AFI, APGAR Score

Article Citation: Aslam I, Mussarat N, Bano S.Perinatal outcome in low ammniotic fluid index. Professional Med J 2015;22(1):137-142.

\section{INTRODUCTION}

In high risk pregnancies, there is a dire need to assess amniotic fluid volume. If values of amniotic fluid volume fall within normal limits, it reassures us regarding well being of fetus. The accurate measurement of amniotic fluid volume can only be done by dye dilution technique or by direct measurement at the time of caesarean delivery. Both are invasive methods. So there was a continuous effort to find out a non invasive method to assess amniotic fluid volume.

Currentlyanestimated lowAFV byultrasound could represent a compromised fetus and generally initiates additional testing, administration of corticosteroids to premature fetus i-e $<34$ weeks and induction with delivery of older pregnancies even if other fetal assessment modalities are reassuring. Additional interventions / delivery are undertaken because low AFV is considered by many clinicians to represent chronic fetal stress and a hostile intrauterine environment which should be best managed by efforts to accomplish delivery. These interventions of induction and delivery might contribute to increased maternal and fetal morbidity rather than a decrease.

The fetal surveillance should effectively identify the fetuses exposed to intrauterine hypoxia and may therefore improve fetal outcome. ${ }^{(1)}$

The ultrasonographic observation of diminished amniotic fluid volume has long been recognized as a predictor of adverse perinatal outcome. (2)

Amniotic fluid index $<5 \mathrm{~cm}$ is associated with fetal distress in labour and increased rate of caesarean section. it is also associated with birth asphyxia, meconium aspiration and cord $\mathrm{pH}<7$ and low APGAR score at the time of delivery . ${ }^{(3)}$

In 1987 Phelan et al described the amniotic fluid 
index as the summation of largest vertical pocket in 4 quadrants. This technique of assessing amniotic fluid volume has become increasingly popular in obstetric practice. ${ }^{(4)}$

The American College of Obstetricians and Gynecologists states that AFI is more accurate and reproducible method of determining abnormalities in amniotic fluid volume than other techniques. ${ }^{(5)}$ The goal of ante partum fetal surveillance is to identify the healthy fetus and fetus at risk of death. ${ }^{(6)}$

An AFI < $5 \mathrm{~cm}$ consistent with most ultrasonographic criteria for oligohydramnios has been used as an indication for delivery of infants at or near term. This practice has suggested by Rutherford et al and by sarno et al, who noted a significantly higher risk of caesarean delivery for fetal distress and low APGAR scores for those parturients with an AFI $<5 \mathrm{~cm}$ than for those with AFI $>5 \mathrm{~cm} .{ }^{(7)}$ Since these initial publication, other investigators have not consistently confirmed the association of adverse peripartum outcome with $\mathrm{AFI}<5 \mathrm{~cm}$.

Because oligohydramnios has been circumstantially associated with a variety of ominous pregnancy outcomes such as perinatal death, fetal distress in labour and poor infant condition at birth, obstetricians has increasingly resorted to inducation of labour or antepartum testing of fetal health in pregnancy complicated by decreased amniotic fluid volume. Such widespread application of sonogram derived estimates of amniotic fluid volume inevitably raises concerns that such information might provoke unnecessary interventions.

In order to understand and relate the complex issues regarding AFV, the techniques to assess AFV by ultrasound and correlation of these estimates with actual AFV as well as pregnancy outcome will be reviewed using available published literature.

We sought to assess significance of AFI for pregnancy outcome.
In view of the unusually large number of booked patients through OPD, this study became desirable with the aim of having an insight into mode of delivery and fetal outcome, using AFI as a screening procedure to determine the fetal well being in this group of patients.

\section{Objective:}

To evaluate decreased amniotic fluid index as a reliable predictor of neonatal outcome in terms of APGAR score.

\section{MATERIAL \& METHODS}

\section{Settings:}

Obstetrics and Gynaecology department of Fatima Memorial Hospital, Lahore

\section{Sample Size:}

60 patients diagnosed ultrasonically as oligohydramnios.

\section{Duration of study:}

6 month from 7-2-2007 to 7-8-2007

\section{Sampling Technique:}

Purposive non-probability sampling

\section{Inclusion Criteria:}

All booked singleton pregnant woman between $37-41$ weeks of gestation with AFI $<7 \mathrm{~cm}$ were included in study.

\section{Exclusion Criteria:}

- Patients with ruptured membranes

- $\mathrm{APH}$

- Fetus with gross congenital anomalies

\section{Study Design:}

Descriptive Study

\section{Data Colletion}

All pregnant women, meeting the inclusion criteria admitted in labour room either through emergency or OPD were selected.

All patients who presented with clinical evidence of reduced liquor at gestational age 37 to $41 \mathrm{wks}$ and with sluggish fetal movements underwent 
measurement of amniotic fluid were included in study.

These 60 patients were evaluated with detailed history (age, parity, LMP, relevant past obstetrical history) and then clinical examination was done.

Amniotic fluid index was recorded and these patients were followed till the time of delivery. Those patients who went into spontaneous labour and those who were induced were vigilantly monitored during labour and at any signs of fetal distress immediate caesarean section was performed.

The patients having very low amniotic fluid index and non-reactive CTG were delivered by immediate caesarean section. The expected outcome and associated long term morbidity was explained to the couple. Mode of delivery was noted.

APGAR score of the newborn was noted at one and five minute and those babies having poor APGAR score were resuscitated immediately and shifted to new born nursery and were followed, day by day, till one week after delivery.

\section{Data Analysis:}

The collected information was entered in the SPSS version 10 and analyzed through its statistical package. The variable of demography, (age, duration of pregnancy) were presented as descriptive statistics showing mean, standard deviation and frequency tables. The past history and outcomes of previous pregnancies were presented as proportions. The variables in current pregnancy, its symptoms, duration were presented as continuous data with mean and standard deviation. APGAR score was also listed in class intervals. A scatter diagram was prepared to assess any association. A co efficient of correlation (as value) was calculated. The value of AFI was thus assessed for strength of association between the two indicators, the quantitative measurement were subjected to t-test and qualitative to chi square test. A p value of 0.05 or less was taken as significant.
RESULTS:

This was a descriptive study conducted at Fatima Memorial Hospital, Lahore form $7^{\text {th }}$ February 2007 to $7^{\text {th }}$ August 2007 over a period of 6 months. During this period 60 patients between gestational ages of 37-41 weeks diagnosed ultrasonically as oligohydramnios were included in the study. Most $(75 \%)$ of the patients were in the age group of $21-30$ years and $20 \%$ of the females were in the age group of $31-40$ years, $5 \%$ in the age of less than 20 years. (Table I)

In total 60 patients included in the study, 47 patients had gravidity between $G_{1}-G_{3}, 12$ patients had gravidity $G_{4}-G_{6}$ and only one patient was $\mathrm{G}_{6}$. (Table II )

Among total patients $53 \%$ had gestational age between $37-38$ weeks, $40 \%$ had gestational age of $39-40$ weeks and $7 \%$ patients presented at gestational age of $>40$ weeks. (Table III)

Most of the patients with oligohydramnios were admitted for induction of labour (38\%) $25.2 \%$ presented with sluggish fetal movements, $16.6 \%$ presented with labour pains, $13.5 \%$ had complaint of pregnancy induced hypertension and $6.7 \%$ of them presented with post dates pregnancy > 40weeks (Table IV) . Cardiotocography done at the time of admission showed reactive trace in $47 \%$ of patients, non reactive in $30 \%$ and $33 \%$ of patients had decelerations on cardiotocography. (Table V)

In patients with amniotic fluid index $<2 \mathrm{~cm}$ caesarean section was done in $66.6 \%$ of patients. vaginal spontaneous delivery occurred in $33.4 \%$ of them.

When amniotic fluid index was $3 \mathrm{~cm}, 75 \%$ of the patients delivered by c-section, $16.6 \%$ had spontaneous vaginal delivery and $8.4 \%$ had assisted vaginal delivery. Among patient when amniotic fluid index was $4 \mathrm{~cm}, 50 \%$ delivered by c-section, $38.5 \%$ by spontaneous vaginal delivery and $11.5 \%$ had assisted vaginal delivery. When amniotic fluid index was $5 \mathrm{~cm}, 38.5$ had csection, $53.8 \%$ had spontaneous vaginal delivery 
and $7.7 \%$ had assisted delivery.

In patients with amniotic fluid index of $6 \mathrm{~cm}, 33.3 \%$ delivery by c- section $33.3 \%$ had normal vaginal delivery and $33.3 \%$ had assisted delivery. (Table VI) Regarding neonatal outcome, out of total 60 patients, 32 neonates were meconium stained, when AFI was $3-4 \mathrm{~cm}$.

AFI 5-6 cm was associated normal outcome in 23 neonates. Initial resuscitation was done in 2 babies, who were meconium stained and asphyxiated, and in these patients AFI was $3 \mathrm{~cm}$.

Ventilation and nursery admission was required in babies when AFI was $2 \mathrm{~cm}$. Only 1 baby had hypoxic ischemic encephalopathy.(Table VII) Neonatal APGAR at 1 minute showed that out of 60 patients, 42 had 1 minute APGAR $<7,18$ had 1 minute APGAR score $>7$ and when 5 minutes APGAR was recorded, 25 patients had APGAR < 7 and 35 had APGAR $>7$. (Table VIII)

\begin{tabular}{|c|c|c|}
\hline Age (Years) & No. of patients & $\%$ \\
\hline$<20$ & 3 & 5 \\
\hline $21-30$ & 45 & 75 \\
\hline $31-40$ & 12 & 20 \\
\hline Total & 60 & 100 \\
\hline
\end{tabular}

Table-I: Distribution of patients by age

\begin{tabular}{|c|c|c|}
\hline Gravidity & No. of patients & $\%$ \\
\hline G1 - G3 & 47 & 78 \\
\hline G4 - G6 & 12 & 20 \\
\hline$<$ G6 & 1 & 2 \\
\hline Total & 60 & 100 \\
\hline
\end{tabular}

Table-II: Distribution of cases according to Gravidity

\begin{tabular}{|c|c|c|}
\hline Duration (Weeks) & No. of patients & $\%$ \\
\hline $37-38$ weeks & 32 & $53^{\star}$ \\
\hline 39- 40 weeks & 24 & $40^{\star}$ \\
\hline$<40$ weeks & 4 & 7 \\
\hline Total & 60 & 100 \\
\hline $\begin{array}{c}\text { Table-III: Distribution of cases by duration of } \\
\text { pregnancy }\end{array}$ & ${ }^{*}$ p Value $>0.05$ \\
\hline Complaints & No. of patients & $\%$ \\
\hline For induction & 23 & $38^{\star}$ \\
\hline Sluggish fetal movements & 15 & 25.2 \\
\hline Labour pains & 10 & 16.6 \\
\hline PIH & 8 & 13.5 \\
\hline Post dates & 4 & 6.7 \\
\hline Total & 60 & 100 \\
\hline & Table- IV: & \\
\hline Distribution of cases by presenting complaints
\end{tabular}

\begin{tabular}{|c|c|c|}
\hline Results & No. of patients & $\%$ \\
\hline Reactive & 28 & 47 \\
\hline Non-reactive & 18 & 30 \\
\hline Deceleration & 14 & 23 \\
\hline Total & 60 & 100 \\
\hline \multicolumn{2}{|c|}{ Table-V } \\
\hline Distribution of cases by cardiotocography \\
\hline
\end{tabular}

\begin{tabular}{|c|c|c|}
\hline Outcome & No. of patients & $\%$ \\
\hline Meconium stained & 32 & 53.4 \\
\hline Normal & 23 & 38.0 \\
\hline Asphyxiated & 1 & 1.6 \\
\hline $\begin{array}{c}\text { Meconium stained } \\
\text { ventilator assisted }\end{array}$ & 2 & 3.5 \\
\hline $\begin{array}{c}\text { Meconium stained } \\
\text { asphyxiated }\end{array}$ & 2 & 3.5 \\
\hline Total & 60 & 100 \\
\hline \multicolumn{2}{|c|}{ Table - VII: Fetal Outcome } \\
\hline
\end{tabular}

\begin{tabular}{|c|c|c|c|c|c|c|c|}
\hline \multirow{2}{*}{ AFI } & $\begin{array}{c}\text { Number of } \\
\text { subjects }\end{array}$ & \multicolumn{2}{|c|}{ C- Section } & \multicolumn{2}{c|}{ SVD } & \multicolumn{2}{c|}{ Assisted delivery } \\
\hline & Number & $\%$ & Number & $\%$ & Number & $\%$ \\
\hline 3 & 6 & 4 & 66.6 & 2 & 33.41 & 0 & 0 \\
\hline 4 & 12 & 9 & 75 & 2 & 16.6 & 1 & 8.4 \\
\hline 5 & 26 & 13 & 50 & 10 & 38.5 & 3 & 11.5 \\
\hline 6 & 13 & 5 & 38.5 & 7 & 53.8 & 1 & 7.7 \\
\hline & 3 & 1 & 33.3 & 1 & 33.3 & 1 & 33.3 \\
\hline
\end{tabular}




\begin{tabular}{|c|c|c|}
\hline APGAR & No. of patients & $\%$ \\
\hline At I minute & & \\
\hline$<7$ & 42 & 70 \\
\hline$>7$ & 18 & 30 \\
\hline Total & 60 & 100 \\
\hline At 5 minutes & & \\
\hline$>7$ & 25 & 41.7 \\
\hline$<7$ & 35 & 58.3 \\
\hline Total & 60 & 100 \\
\hline Table - VIII: Neonatal APGAR Score \\
\hline
\end{tabular}

\section{DISUSSION:}

Amniotic fluid index is a method of fetal risk surveillance. ${ }^{(5)}$ In 1990 Moore and Cayle developed method of assessment of amniotic fluid volume by dividing the uterus in four quadrants between 16-14 weeks of gestation. Moore in his work designated that AFI is a gold standard for the measurement of AFV. ${ }^{(8)}$ Decreased amniotic fluid index is associated with poor perinatal outcomes. The ideal antepartum test should have high sensitivity and high specificity. Fetal heart rate monitoring is the traditional method of antepartum fetal surveillance. The contraction stress test may be the most sensitive indication of fetal hypoxia. However, the false positive rate may be as high as $40-50 \%$ i.e. low specificity. ${ }^{(9)}$ Where there is poor maternal surveillance, sudden intrauterine fetal death remains one of the most disturbing situation faced not only by the treating obstetrician, but also by the family of the affected patient. This is a common occurrence in developing parts of the world where pregnant women, unaware of the necessity of antenatal care, are seen for the first time in the hospital in late pregnancy or in labour. The timing of delivery of these patients can be quite tasking. The perinatal loss is associated with lack of antenatal care.

This study reviewed the neonatal outcome in patients with oligohydramnios (AFI $<7 \mathrm{~cm}$ ). Most of the patients (75\%) had age group of 21-30 years, although no association of the oligohydrmnios and maternal age had been identified till now.

The gestational age in $53 \%$ of the patients was 37 38 weeks and $40 \%$ of the patients had gestation of
$39-40$ weeks. Only $7 \%$ had $>40$ weeks gestations because most of the obstetrician prefers to deliver the patients when amniotic fluid had decreased either by induction or by caesarean section. So less percentage is seen beyond 40 weeks. Admission CTG showed reactive trace in $47 \%$ patients had non reactive CTG in 53\%. These results are comparable with study by Baron in 1995, which showed that AFI $<5 \mathrm{~cm}$ is associated with increased risk of variable decelerations. ${ }^{(10)}$ Increased caesarean section rate was seen in patient with AFI $3 \mathrm{~cm}, 75 \%$ patients delivered by caesarean section, these results are comparable with study of Chauhan et al, which showed that caesarean section for fetal distress and neonatal APGAR $<7$ at 5 minutes occurred in significant by greater number of patients with AFI $<5 \mathrm{~cm}$. ${ }^{(11)}$ Among 60 patients included in the study, 32 babies (53.4\%) had meconium stained liquor and APGAR scores recorded at 1 minute showed that $70 \%$ of the babies had APGAR $<7$ at I minute which improved by active resuscitation and at 5 minute $41.7 \%$ of the babies had APGAR $<7$ at 5 minute and it is comparable with study of Jeng et al in which AFI $<8 \mathrm{~cm}$ was associated with greater risk of meconium stained amniotic fluid, fetal distress, abnormal fetal heart rate monitoring and APGAR $<7$ at 5 min. ${ }^{(12)}$

In developing parts of the world where poverty, poor patients compliance nutritional deprivation, unsatisfactory system and multiparty have all contributed to late presentation of pregnant women to the hospital and the association can be enormous. A purposeful ultrasonography in conjunction with a non stress test, with a view to elucidate the amniotic fluid index could go a long way to select and expedite delivery in fetuses at risk if such services could be provided. It is important to concentrate on maternal health and antenatal care. ${ }^{(13)}$

\section{CONCLUSION:}

It is concluded from the above study that whenever there are subjective or objective complaints of sluggish fetal movements or clinical suspicion of decreased liquor, AFI should be done to assess the near actual condition of the fetus so that if 
necessary the fetal outcome can be improved by proper intervention. This study showed that the patients having decreased AFI, delivered babies with low APGAR scores. However despite the complaints of decreased fetal movements and clinically small babies most of the patients had normal AFI and the babies delivered with good APGAR score at 5 minute. It means AFI effectively detected those patients who really needed early intervention and thus avoiding unnecessary inductions and caesarean section with related morbidity.

Copyright(C) 09 Jan, 2015.

\section{REFERENCES}

1. Mohsin $\mathrm{H}$ Neonatal outcomes as a predicted by biophysical profile. J Coll Physician Surg Pak 2001.11.149-51

2. Gumus II, Koktenner A, Turhan No. Perinatal outcomes of pregnancies with borderline amniotic fulid index.Arch Gynecol obstet 2007; 276:17-9.

3. Morris JM, Thomposon K, Smithey J, Gaffeny G, Cooke I, Chamberlain P. The usefulness of ultrasound assessment of amniotic fluid in predicting adverse outcomes in prolonged pregnancy. A prospective blinded observational study 2003;110:989-94.

4. Brace RA Wolf EJ Normal amniotic fluid volume changes throughout pregnancy. AM J Obstet Gynecol 1989;161:382-8.

5. Moore TR. Cayle JE.The amniotic fluid index in normal human pregnancy. AM J Obstet Gynecol 1990;162:116873.

6. Dayal Ak, Manning FA, Berck DJ Mussalli GM, Aliva C, Harman CR, et al.Fetal death after normal biophysical profile score. An eighteen year experience. AM J Gynecol 2000;183:783.

7. Seeds AE Current concepts of amniotic Fluid dynamics. AM J Obstet Gynecol 1980;138:575-86.

8. Moore TR. Superiority of four quardrant sum over the signal deepest pocket techique in ultrassonographic identification of abnormal amniotic fulid volume. AM J Obstet Gynecol 1990;162:1168-73.

9. Braly P, Freeman RK. The significance of fetal heart rate reactivity with a positive oxytocin challenge test. Obs Gynae 1977;50:689-91.

10. Baron C, Morgan MA, Garite TJ. The impact of amniotic fluid volume assessed intrapaartum on perinatal outcome. Am Obstet Gynecol 1995;173:167-74.

11. Chauhan SP, sanderson M, Hendrx NW, Magann EF, Devoe LD. Perinatal outcome and amniotic fluid index in antepartum and intrapartum periods. AM J Obstet Gynecol 1999;181:1478.

12. Jeng $\mathrm{Cj}$, Jou TJ, Wang KG, Yang, YC, Lee YN, Lan, CC. Amniotic fluid index measurement with four quadrant technique during pregnancy. J Reprod Med 1990;35:674-7.

13. Ejaz I, Khan IH, Baloch RG. Neonatal mortality Report from a tertiary hospital in Lahore / causes and outcomes. Pak Pead J 2001;25:35-8. 